\title{
Révision des effets de l'amidon et des protéines du régime sur l'évacuation gastrique chez le porc
}

\author{
J.P. LAPLACE, Odile PONS * et P. SIMON* \\ I.N.R.A., Laboratoire de Physiologie de la Nutrition \\ * Laboratoire de Biométrie \\ Centre de Recherches zootechniques, F 78350 Jouy-en-Josas
}

\begin{abstract}
Résumé
L'évacuation gastrique de 4 régimes formulés à 16 p. 100 de matières azotées, et résultant de la combinaison factorielle de 2 amidons purifiés (maïs ou blé) et de 2 sources protéiques (farine de poisson ou gluten de blé) a été antérieurement décrite (LAPLACE et al., 1981) sur la base de la mesure des contenus gastriques résiduels à divers temps postprandiaux chez 12 porcs équipés d'une canule gastrique permanente. L'analyse mathématique de ces résultats descriptifs de l'évacuation de la matière fraîche, de la matière sèche, de l'amidon et de l'azote, est remise en question. Il s'avère que, pour les 4 variables contrôlées, le facteur temporel de répétition des épreuves d'une semaine à l'autre est nul et que pour 3 de ces variables (azote excepté), la valeur des observations réalisées à chaque instant de la cinétique est indépendante des facteurs temporels. Aussi, les analyses de variance effectuées reposent sur un modèle univariate à données scalaires, sauf dans le cas de l'azote pour lequel est utilisé un modèle multivariate à données vectorielles.

Il ressort de cette ćtude que (i) la nature de l'amidon est en elle-même sans influence dans le cas des amidons purifiés utilisés; (ii) que l'association particulière d'un amidon à une protéine affecte le niveau moyen d'évacuation de la matière fraîche et de l'amidon, mais que celui de la matière sèche reste exempt de tout effet du régime; (iii) que la source de protéines alimentaires influence fortement le déroulement de l'évacuation de la matière fraîche, de la matière sèche et de l'amidon. L'importance de la distinction des effets exercés à l'égard du niveau moyen d'évacuation d'une part, et du déroulement de celle-ci d'autre part, est soulignée. L'association particulière d'un amidon et d'une protéine affecterait le niveau moyen d'évacuation, et de ce fait la durée globale de digestion du repas; les protéines joueraient un rôle décisif à l'égard du déroulement de l'évacuation et donc à l'égard de la cinétique de mise à disposition ultérieure des nutriments. Enfin l'influence de l'animal s'avère considérable vis-à-vis du niveau moyen d'évacuation, et masque les effets du régime sur l'évacuation gastrique de l'azote.
\end{abstract}

\section{Introduction}

L'expérimentation chez l'animal fistulé à divers niveaux du tube digestif, pour évaluer le transit et la digestion des matériaux alimentaires dans l'estomac et/ou l'intestin grêle se heurte classiquement à divers problèmes liés d'une part à la méthodologie d'étude de ces phénomènes et d'autre part à la durée de vie expérimentale 
de certaines préparations face aux besoins expérimentaux : utilisation comparative de plusieurs régimes, nécessité de périodes d'accoutumance aux aliments. Le premier de ces problèmes tient à la nature même d'une étude de cinétique de digestion d'un repas, reposant sur des collectes consécutives de quantités qui ne sont d'évidence pas indépendantes. Aussi, l'influence du temps est-elle susceptible d'affecter l'analyse des facteurs expérimentaux étudiés (GILL \& HAFS, 1971). Ces observations conduisent à l'usage des procédures multidimensionnelles (KrishNAIAH, 1969) pour tenir compte de cette dépendance en étudiant l'ensemble des variables $\left(\mathrm{y}_{\left(\mathrm{t}_{1}\right)}, \ldots, \mathrm{y}_{\left(\mathrm{t}_{\mathrm{n}}\right)}\right)$ c'est-à-dire des collectes effectuées aux divers instants (LAPLACE et al, 1981). Le second problème tient à l'effet de l'animal dans la mesure où il n'est pas matériellement possible de tester tous les régimes chez tous les animaux en multipliant les jours d'épreuve, en réservant les périodes d'accoutumance, et en prenant en compte l'ordre de passage des divers aliments étudiés (LAPlace, Darcy, Pons, 1983).

L'évaluation de l'effet de l'animal dans le cas fréquent où chaque régime est étudié sur un groupe différent d'individus, passe donc classiquement par l'étude d'un effet d'interaction régime-animal. L'existence d'un tel effet significatif d'interaction a ainsi été mise en évidence à l'égard de certaines des variables prises en compte pour l'étude de la digestion dans l'intestin grêle chez le porc (Darcy, Laplace \& Villiers, 1981). Ceci revient à dire que l'introduction du facteur porc en tant que tel dans l'écriture du modèle d'analyse de variance, comme cela a été fait dans une première étude par analyse multidimensionnelle de l'évacuation gastrique chez le porc (LAPLACE et al., 1981) n'est pas véritablement licite. Une telle écriture du modèle a pour conséquence de réduire la résiduelle, et partant de surestimer les effets testés.

Il découle de ces observations d'ordre méthodologique que l'importance réelle de certains des effets antérieurement décrits (LAPLACE et al., 1981) de la nature de l'amidon et de la source de protéines alimentaires sur l'évacuation gastrique chez le porc a pu être surestimée. L'objet du présent travail est donc de réévaluer leur signification effective.

\section{Matériel et méthodes}

\section{A. Rappel de l'expérience}

L'étude expérimentale concernée repose sur la collecte des contenus résiduels dans l'estomac de porcs préalablement équipés avec une canule gastrique. Ces collectes sont effectuées à divers temps $(30 \mathrm{mn}, 1,2,4$ ou $7 \mathrm{~h})$ après ingestion par les animaux de quantités connues de l'un ou l'autre des 4 régimes résultant de la combinaison factorielle de 2 amidons purifiés (de blé ou de maïs) et de 2 protéines (farine de poisson ou gluten de blé). Les quantités résiduelles de matière fraîche, matière sèche, amidon et azote sont déterminées dans les contenus gastriques recueillis. On en déduit les quantités correspondantes déjà évacuées par l'estomac (les 4 variables contrôlées) aux divers instants de mesure. Les modalités d'alimentation des porcs et la composition détaillée des régimes, la méthodologie de collecte, d'échantillonnage et d'analyse des contenus gastriques, ainsi que les résultats obtenus, ont été décrits antérieurement (LAPLACE et al., 1981). Ces éléments descriptifs de l'évacuation gastrique ont déjà fait l'objet (i) d'une analyse de variance multidimensionnelle dont le 
modèle intègre un effet propre du porc, puis (ii) d'une modélisation par ajustement polynomial des données (LAPLACE et al., 1981).

\section{B. Inventaire des facteurs}

Dans le dispositif expérimental mis en œuvre, 12 porcs ont été affectés au hasard, à raison de 3 porcs $(\mathrm{p}=1,2,3)$ par groupe, à l'un ou l'autre des 4 régimes $(\mathrm{r}=1, \ldots, 4)$ correspondant aux combinaisons (schéma factoriel) des 2 types d'amidon $(i=1,2)$ et des 2 types de protéines $(j=1,2)$. Ainsi chaque animal ne fournit de mesures que dans le cas d'un seul couple amidon-protéine. Par ailleurs, une seule collecte des contenus gastriques est effectuée pour chaque jour d'épreuve, ce qui revient à dire que la constitution d'une «cinétique » (5 temps) pour chacune des 4 variables contrôlées s'effectue en 5 jours, et que 3 cinétiques sont obtenues pour un régime donné chez un même animal en 3 semaines.

La valeur des observations peut donc être affectée par divers facteurs, les uns temporels, les autres liés au régime et aux porcs. Ainsi, la semaine et le jour d'épreuve sont des facteurs temporels parasites. On peut valablement admettre a priori l'indépendance des mesures d'une semaine à l'autre. Par ailleurs, toute mesure effectuée un jour donné fait partie de l'ensemble des observations de la semaine en cours. Les facteurs semaine et jour sont donc hiérarchiques. En ce qui concerne enfin la durée postprandiale préalable à la collecte des contenus gastriques, on note que l'ensemble des durées est isomorphe à l'ensemble des jours. Seuls seront donc en définitive considérés les facteurs semaine $(\mathrm{s}=1,2,3)$ et durée $(\mathrm{d}=1, \ldots, 5)$. La valeur des observations est encore affectée simultanément par les animaux sur lesquels elles ont été réalisées et par le régime alimentaire auquel ils ont été assignés. L'effet aléatoire du porc est ainsi lié à l'effet du régime, et l'écriture du modèle d'analyse de variance comprendra donc deux termes distincts exprimant l'un l'effet régime et l'autre l'effet hiérarchique régime/porc.

Enfin, du fait de la variabilité des réponses, à la fois entre les porcs et entre les mesures effectuées chez un même animal, les modèles d'analyse de variance seront des modèles «en blocs» (SCHEFFE, 1967). Dans ces modèles, chaque porc est considéré comme un bloc sur lequel est appliqué un traitement (régime) donné. Les différentes semaines, et éventuellement les divers jours d'épreuve constituent alors des sousblocs.

\section{Résultats}

\section{A. Modélisation}

Un modèle satisfaisant, compte tenu de l'hypothèse initialement admise de l'indépendance des mesures d'une semaine à l'autre, est constitué par 36 vecteurs données $\vec{Y}_{\text {rps }}$ ( 4 régimes $\times 3$ porcs $\times 3$ semaines) de taille 5 , c'est-à-dire comportant chacun les 5 mesures effectuées sur un porc donné affecté à un régime (r, p) au cours d'une semaine donnée (s). Dans ce modèle multivariate, en l'absence a priori de toute 
additivité entre les facteurs ( $r, p)$ et (s), chaque donnée peut être décomposée de la façon suivante :

$$
\overrightarrow{\mathrm{Y}}_{\mathrm{rps}}=\left(\mathrm{a}+\mathrm{b}_{\mathrm{r}}+\mathrm{I}_{\mathrm{rp}}+\mathrm{e}_{\mathrm{s}}+\mathrm{f}_{\mathrm{rs}}+\mathrm{K}_{\mathrm{rps}}\right) 1, \ldots, 5
$$

On notera que la durée est ici une dimension du modèle multivariate et qu'elle n'intervient donc pas comme un facteur.

Un modèle plus simple consiste cependant à supposer toutes les mesures indépendantes, et à considérer des observations scalaires $Y_{\text {rpsil }}$ (4 régimes $\times 3$ porcs $\times 3$ semaines $\times 5$ durées, soit 180 mesures). Dans un tel modèle univariate, la durée postprandiale (isomorphe aux jours) est donc prise en compte comme un facteur.

Face à ces deux modèles plausibles, il convient de choisir, pour chacune des variables contrôlées, celui d'entre eux qui est le mieux adapté. Il s'agit en d'autres termes de tester les liens entre les mesures effectuées aux différentes durées. Le choix entre les modèles multivariate et univariate peut être réalisé en testant la matrice de variance-covariance de $\vec{Y}$ par le maximum de vraisemblance. Par rapport à un seuil de rejet de 1 p. $100(\mathrm{~L}>27,69)$ la statistique du rapport de vraisemblance, qui suit une loi de $\chi^{2}$ à 13 degrés de liberté, fournit les valeurs suivantes : matière fraîche, $\mathrm{L}=24,46$; matière sèche, $\mathrm{L}=25,12$; amidon, $\mathrm{L}=27,16$; azote, $\mathrm{L}=41,93$. Ceci signifie donc que : (i) le modèle univariate à données scalaires est adéquat pour l'étude de la matière fraîche, de la matière sèche et de l'amidon; (ii) le modèle multivariate à données vectorielles est nécessaire pour l'étude de l'azote. Dans les 2 cas, il s'agit de modèles en blocs mixtes comportant des effets fixes (régime, semaine, durée postprandiale) et un effet aléatoire (porc).

\section{B. Matière fraîche, matière sèche et amidon : réduction du modèle univariate}

Le modèle étudié pour ces 3 variables contient donc 180 données scalaires dont les valeurs sont conditionnées, comme il a été exposé plus haut, par les facteurs régime et porc (indicés $r$ et $r p$ ), durée postprandiale (indicée d), semaine et jour (indicés $s d$, du fait de leur hiérarchie et de l'isomorphisme des jours et des durées). L'écriture du modèle résultant du croisement de ces divers termes est donc la suivante :

(1) $Y_{r p s d}=a+b_{r}+I_{r p}+c_{d}+d_{r d}+J_{r p d}+e_{s}+f_{r s}+K_{r p s}+g_{s d}+h_{r s d}+L_{r p s d}$ où les termes aléatoires $I_{r p}, J_{r p d}, K_{r p s}$ et $L_{\text {rpsd }}$ seront considérés comme des variables gaussiennes, indépendantes deux à deux.

L'examen des sommes de carrés pour chacun des termes de ce modèle (1) suggère que le facteur semaine pourrait être un authentique facteur de répétition. L'hypothèse de nullité des termes $e_{\mathrm{s}}, f_{\mathrm{rs}}, g_{\mathrm{sd}}, h_{\mathrm{rsd}}$ et $K_{\mathrm{rps}}$ peut être testée par le test $F$ de Fisher. La région de rejet de l'hypothèse au seuil de 5 p. 100 est $F_{1}>1,54$. Les valeurs de $F_{1}$ enregistrées pour la matière fraîche, la matière sèche et l'amidon, sont de $1,19,1,46$ et 1,26 respectivement. Par conséquent, pour ces 3 variables contrôlées, on vérifie que les effets liés à la semaine, initialement supposés indépendants, peuvent en fait être considérés comme nuls. Le modèle (1) peut valablement être simplifié, et s'écrit alors :

$$
\mathrm{Y}_{\mathrm{rpsd}}=\mathrm{a}+\mathrm{b}_{\mathbf{r}}+\mathrm{I}_{\mathrm{rp}}+\mathrm{c}_{\mathrm{d}}+\mathrm{d}_{\mathrm{rd}}+\mathrm{J}_{\mathrm{rpd}}+\mathrm{L}_{\mathrm{rpsd}}
$$

Ce modèle (2) liste donc, pour les variables matière fraîche, matière sèche et 
amidon, les facteurs exerçant un effet sur l'évacuation gastrique dans les conditions expérimentales utilisées. Chacun des termes revêt en outre une signification particulière :

- Par rapport à une valeur moyenne (terme a), l'addition du terme $c_{d}$ permet de déterminer une courbe moyenne générale d'évacuation gastrique au fil du temps postprandial.

- L'introduction du terme $b_{r}$ en plus des deux précédents $\left(a+c_{d}\right)$ fait apparaître la différence due en moyenne à l'effet de tel ou tel régime. Le terme $b_{r}$ conduit à une simple translation par rapport à la courbe moyenne générale, sans affecter le profil d'évacuation.

- C'est l'addition supplémentaire du terme d'interaction régime-durée $\left(\mathrm{d}_{\mathrm{rd}}\right)$ qui va affecter le profil de la courbe pour fournir la description du décours de l'évacuation caractéristique d'un régime testé sur un groupe de porcs.

- Par rapport à cette dernière courbe $\left(a+c_{a l}+b_{r}+d_{r d}\right)$, une translation rend compte de la variation moyenne due au porc lorsqu'est ajouté le terme $\mathbf{I}_{\mathrm{rp}}$.

- Le terme $\mathbf{J}_{\mathbf{r p d}}$ introduit seulement une variation ponctuelle de la courbe d'évacuation, c'est-à-dire une particularité de l'évacuation d'un régime chez un porc à l'un des instants d'observation.

- Enfin, la résiduelle $\mathrm{L}_{\text {rpsd }}$ introduit toute autre source de variation.

C. Matière fraîche, matière sèche et amidon : estimations et tests dans le modèle univariate simplifié

Avant de procéder aux estimations des paramètres dans le modèle (2), il convient de substituer à l'indice $\mathbf{r}$ du régime, les indices $\mathrm{i}$ et $\mathrm{j}$ correspondant respectivement à la nature de l'amidon et à la source de protéines qui entrent dans la composition de l'aliment expérimental. Le modèle univariate simplifié s'écrit alors :

$$
Y_{i j p s d}=a+e_{i}+f_{j}+g_{i j}+I_{i j p}+c_{d}+m_{i, l}+n_{j u l}+o_{i j d}+J_{i j p d}+L_{i j p s d}
$$

Dans le cas de chacune des 3 variables contrôlées, étudiées selon ce modèle, le calcul fournit les estimations des divers termes, à partir desquelles peuvent être construites des courbes moyennes d'évacuation (fig. 1) pour chaque régime. Chacune de ces courbes représente la sommation des effets fixes suivants :

$$
a+e_{i}+f_{j}+g_{i j}+c_{d}+m_{i d}+n_{j d}+o_{i j d}
$$

Par ailleurs, les rapports des carrés moyens pour ces divers termes au carré moyen résiduel (variance ratio, V.R.) sont les valeurs de statistiques qui suivent une loi de Fisher et permettent donc de tester l'éventualité du caractère négligeable des effets exprimés par ces termes. Les résultats de ces tests sont rassemblés, respectivement pour la matière fraîche, la matière sèche et l'amidon, dans les tableaux 1 à 3 .

\section{Matière fraîche (tabl. 1)}

Lorsqu'on teste l'hypothèse de nullité des divers termes du modèle, il apparaît qu'en lui-même l'effet de la nature de l'amidon $\left(\mathrm{e}_{\mathrm{j}}\right)$ est négligeable, alors que les effets de la source de protéines $\left(f_{j}\right)$ et de l'interaction amidon-protéine $\left(g_{i j}\right)$ sont importants. Toutefois, si l'effet principal de l'amidon est négligeable, il ne peut être considéré 


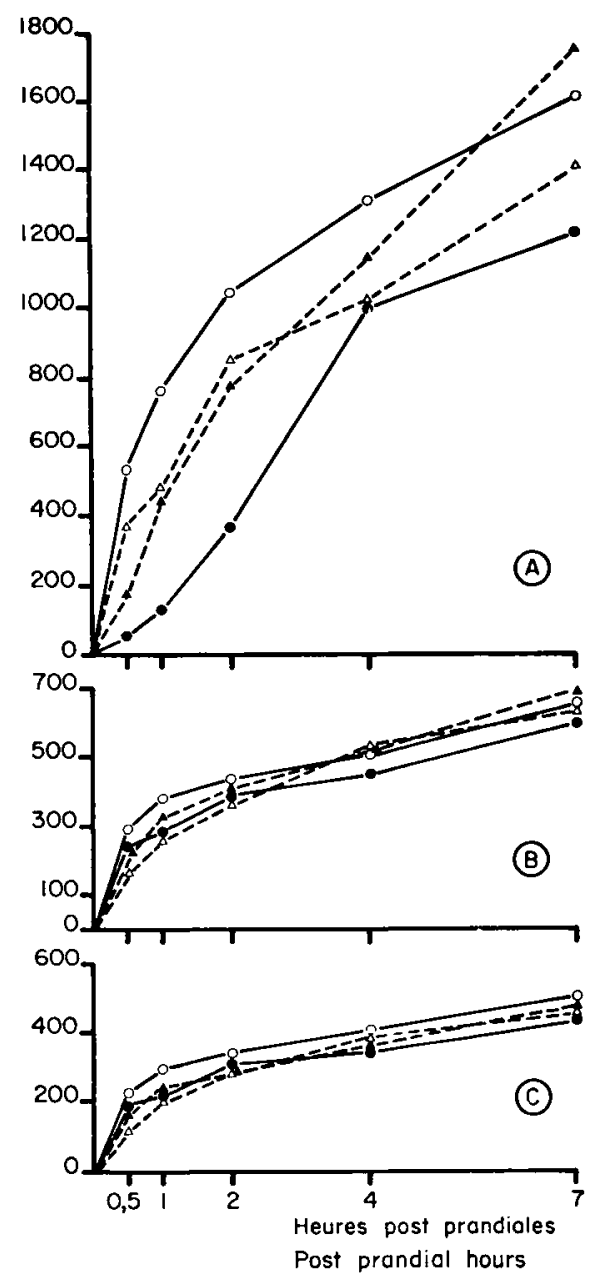

FIG. 1

Courbes moyennes d'évacuation gastrique (quantités en g) de la matière fraîche $(A)$, de la matière sèche $(B)$ et de l'amidon $(C)$ selon la nature du régime.

Ces courbes résultent de l'estimation des divers termes exprimant les seuls effets fixes (amidon, protéine, durée postprandiale et leurs interactions) du modèle (3).

Mean gastric emptying curves (quantities as $g$ ) of wet digesta $(A)$, dry matter $(B)$ and starch $(C)$ according to the diet.

These curves result from the estimation of the various terms representative of the fixed effects only (starch, protein, postprandial duration, and their interactions) in the model (3).

O $\quad$ Amidon de maïs-gluten de blé.

Maize starch-wheat gluten.

- - Amidon de maïs-farine de poisson.

Maize starch-fish meal.

$\triangle-\cdots-\triangle$ Amidon de blé-gluten de blé.

Wheat starch-wheat gluten.

A...... Amidon de blé-farine de poisson.

Wheat starch-fish meal. 
comme absolument nul puisque l'effet $\left(\mathrm{e}_{\mathrm{i}}+\mathrm{g}_{\mathrm{ij}}\right)$ est significatif. Ceci revient à dire que la source de protéines du régime exerce un effet important à l'égard de l'évacuation gastrique, mais que cet effet est toujours significativement influencé par la nature de lamidon associé aux protéines alimentaires. Au total, l'effet du régime (couple amidon-protéine) sur les quantités de matière fraîche évacuées en moyenne par l'estomac est donc important $\left(e_{i}+f_{j}+g_{i j}\right)$. Ainsi l'évacuation de la matière fraîche est-elle en moyenne plus élevée pour l'association d'un amidon de maïs et d'un gluten de blé, et la plus faible pour le régime amidon de maïs-farine de poisson.

TABLEAU 1

Facteurs de variation de l'évacuation gastrique de la matière frâtche.

Sources of variation in the gastric emptying of wet digesta.

\begin{tabular}{|c|c|c|c|c|}
\hline $\begin{array}{l}\text { Terme étudié } \\
\text { Studied term }\end{array}$ & $\begin{array}{l}\text { Loi de VR } \\
\text { Law of } V R\end{array}$ & $\begin{array}{l}\text { Rejet de nullité } \\
\text { Nullity rejection }\end{array}$ & $\begin{array}{l}\text { Valeurs de VR } \\
\text { Values of VR }\end{array}$ & $\begin{array}{l}\text { Effets significatifs } \\
\text { Significant effects }\end{array}$ \\
\hline$e_{i}$ & F $(1,8)$ & $\mathrm{VR}>5,318$ & 0,217 & - \\
\hline$f_{j}$ & F $(1,8)$ & $\mathrm{VR}>5,318$ & 8,319 & $* *$ \\
\hline$g_{i j}$ & $F(1,8)$ & $\mathrm{VR}>5,318$ & 10,773 & $* *$ \\
\hline$e_{i}+g_{i j}$ & $\mathrm{~F}(2,8)$ & $V R>4,459$ & 5,500 & $* *$ \\
\hline$f_{j}+g_{i . j}$ & F $(2,8)$ & $V R>4,459$ & 9,546 & $* * *$ \\
\hline $\mathrm{e}_{\mathrm{i}}+\mathrm{f}_{\mathrm{j}}+\mathrm{g}_{\mathrm{ij}}$ & F $(3,8)$ & $\mathrm{VR}>4,066$ & 6,436 & $* *$ \\
\hline$c_{d}$ & $F(4,32)$ & $V R>2,670$ & 153,238 & $* *$ \\
\hline $\mathrm{m}_{\mathrm{i} i \mathrm{i}}$ & $\mathrm{F}(4,32)$ & $\mathrm{VR}>2,670$ & 1,499 & - \\
\hline $\mathrm{n}_{j, 1}$ & $F(4,32)$ & $V R>2,670$ & 4,182 & $* *$ \\
\hline $\mathrm{o}_{\mathrm{i}, \mathrm{j} !}$ & $F(4,32)$ & $\mathrm{VR}>2,670$ & 1,133 & 一 \\
\hline$m_{i, l}+c_{i j, l}$ & $F(8,32)$ & $\mathrm{VR}>2,250$ & 1,316 & 一 \\
\hline $\mathbf{m}_{i, 1}+\mathbf{n}_{j, 1}+o_{i, j d}$ & $F(12,32)$ & $\mathrm{VR}>2,070$ & 2,271 & $* *$ \\
\hline $\mathbf{J}_{\mathrm{i}, j \mu \mathrm{l}}$ & $F(32,120)$ & $V R>1,540$ & 1,180 & - \\
\hline$I_{i j p}+J_{i, j p c l}$ & $F(40,120)$ & $V R>1,495$ & 2,152 & $* *$ \\
\hline
\end{tabular}

Valeurs du variance ratio (VR) pour chacun des termes ou groupes de termes du modèle (3). La région de rejet de l'hypothèse de nullité de l'effet considéré est indiquée au seuil de 5 p. 100.

Values of the variance ratio (VR) for each term or group of terms in model (3). The rejection of the hypothesis of nullity of the considered effect is indicated at the level of $5 \mathrm{p} .100$.

Par ailleurs, si l'effet de la durée postprandiale $\left(c_{t}\right)$ est évidemment très significatif (il correspond au décours de l'évacuation), on constate que seul l'effet d'interaction protéine-durée $\left(\mathrm{n}_{\mathrm{jd}}\right)$ est important. En d'autres termes, le profil des courbes ou décours de l'évacuation n'est affecté que par la source de protéines du régime. Aussi, pour une protéine donnée, les deux courbes moyennes obtenues selon la nature de l'amidon 
qui lui est associé, sont parallèles. L'évacuation gastrique de la matière fraîche est ainsi nettement plus rapide pour les régimes gluten au cours des premières heures postprandiales (courbes plus pentues à l'origine). Au total, le régime (couple amidonprotéine) affecte significativement non seulement la valeur moyenne des quantités de matière fraîche évacuées, mais aussi le profil des courbes d'évacuation $\left(m_{i d}+n_{j d}\right.$ $\left.+o_{i j d}\right)$.

En ce qui concerne le facteur aléatoire porc, la nullité de l'effet $J_{i j p d}$ signifie que le porc n'affecte pas le profil des courbes. Mais l'interaction régime-porc $\left(\mathrm{I}_{\mathrm{ijp}}\right)$ significative implique des quantités moyennes évacuées différentes pour un même régime selon le porc utilisé. En d'autres termes, le facteur porc introduit une simple translation de la courbe moyenne pour le régime concerné, sans affecter le décours de l'évacuation de l'estomac. Au demeurant, si on teste globalement l'ensemble des termes pour lesquels l'hypothèse de nullité a été vérifiée individuellement, on constate que $\operatorname{VR}\left(\mathrm{m}_{\mathrm{i} l}+\mathrm{o}_{\mathrm{ijl}}+\mathrm{J}_{\mathrm{ijpl}}\right) \simeq \mathrm{F}_{(40,120)}=1,254$, ce qui confirme la nullité de ces termes d'interaction avec la durée postprandiale (rejet de nullité pour VR $>1,495$ ).

\section{Tableau 2}

Facteurs de variation de l'évacuation gastrique de la matière sèche (principe de ce tableau identique à celui du tableau 1).

Sources of variation in gastric emptying of dry matter (for explanation, see table 1).

\begin{tabular}{|c|c|c|c|c|}
\hline $\begin{array}{l}\text { Terme étudié } \\
\text { Studied term }\end{array}$ & $\begin{array}{l}\text { Loi de VR } \\
\text { Law of } V R\end{array}$ & $\begin{array}{l}\text { Rejet de nullité } \\
\text { Nullity rejection }\end{array}$ & $\begin{array}{l}\text { Valeurs de VR } \\
\text { Va!ues of VR }\end{array}$ & $\begin{array}{l}\text { Effets significatifs } \\
\text { Significant effects }\end{array}$ \\
\hline$e_{i}$ & $F(1,8)$ & $\mathrm{VR}>5,318$ & 0,190 & 一 \\
\hline $\mathbf{f}_{\mathrm{j}}$ & $F(1,8)$ & $\mathrm{VR}>5,318$ & 0,064 & 一 \\
\hline $\mathrm{g}_{\mathrm{ij}}$ & $\mathrm{F}(1,8)$ & $\mathrm{VR}>5,318$ & 6,265 & $* *$ \\
\hline $\mathrm{e}_{\mathrm{i}}+\mathrm{f}_{\mathrm{j}}+\mathrm{g}_{\mathrm{ij}}$ & $F(3,8)$ & $V R>4,066$ & 2,173 & - \\
\hline $\mathrm{c}_{\mathrm{l}}$ & $\mathrm{F}(4,32)$ & $V R>2,670$ & 321,280 & $* *$ \\
\hline $\mathrm{m}_{\mathrm{id}}$ & $F(4,32)$ & $\mathrm{VR}>2,670$ & 1,111 & - \\
\hline $\mathbf{n}_{\mathrm{jd}}$ & $F(4,32)$ & $\mathrm{VR}>2,670$ & 8,206 & $* *$ \\
\hline$o_{i j d}$ & $F(4,32)$ & $\mathrm{VR}>2,670$ & 1,240 & - \\
\hline$m_{i d l}+o_{i j !}$ & $\mathrm{F}(8,32)$ & $\mathrm{VR}>2,250$ & 1,175 & 一 \\
\hline $\mathrm{m}_{\mathrm{i}, l}+\mathrm{n}_{\mathrm{j}, \mathrm{l}}+\mathrm{o}_{\mathrm{j}, \mathrm{d} d}$ & $F(12,32)$ & $\mathrm{VR}>2,070$ & 3,519 & $* *$ \\
\hline $\mathrm{J}_{\mathrm{ijpd}}$ & $F(32,120)$ & $\mathrm{VR}>1,540$ & 1,356 & 一 \\
\hline$I_{i j p}+J_{i j p d}$ & $F(40,120)$ & $V R>1,495$ & 3,258 & $* *$ \\
\hline
\end{tabular}

2. Matière sèche (tabl. 2)

Dans le cas de la matière sèche, aucun des 2 facteurs amidon $\left(e_{i}\right)$ et protéine $\left(f_{j}\right)$ ne paraît exercer une influence significative. Bien que l'effet de leur interaction ne 
soit pas nul, il s'avère que l'effet global du régime (couple amidon-protéine; $e_{i}+f_{j}$ $+g_{i j}$ ) est nul. En somme, aucune des 4 combinaisons amidon-protéine étudiées ne conduit en moyenne à des quantités évacuées différentes des autres.

L'influence $d u$ facteur durée postprandiale $\left(c_{1}\right)$ est d'évidence là encore significative. Mais l'examen des valeurs de VR pour les termes d'interaction avec la durée $\left(m_{\mathrm{il}}, \mathbf{n}_{\mathrm{jd}}, \mathrm{o}_{\mathrm{ija}}\right)$ montre que l'influence de la nature de l'amidon est nulle à ce niveau, ce que vérifie $\mathrm{VR}\left(\mathrm{m}_{\mathrm{ill}}+\mathrm{o}_{\mathrm{ijd}}\right)$, alors que celle de la source de protéines $\left(\mathrm{n}_{\mathbf{j} \mathrm{l}}\right)$ est bien réelle. Au total, l'influence du régime sur le profil des courbes d'évacuation $\left(\mathrm{m}_{\mathrm{id}}+\mathrm{n}_{\mathrm{jd}}+\mathrm{o}_{\mathrm{ijd}}\right)$ est significative. De ces éléments on peut donc retenir que, si les 4 régimes sont en moyenne équivalents, la source de protéines affecte de façon non négligeable le décours de l'évacuation gastrique de la matière sèche (profil des courbes).

Enfin, par rapport à ces effets au total limités du régime, l'influence du facteur porc est relativement importante. Comme dans le cas de la matière fraîche, l'effet de l'animal n'introduit pas de modification du décours de l'évacuation de la matière sèche mais une simple translation par rapport à la courbe moyenne pour les régimes concernés. Là encore, le test global pour les termes $\left(m_{i 1 l}, o_{i j d i}, J_{i j, j l}\right)$ confirme la nullité de ces termes d'interaction avec la durée $\left(\mathrm{VR} \simeq \mathrm{F}_{(41.120)}=1,404\right.$; rejet pour VR $>1,495$ ).

\section{TABLEaU 3}

Facteurs de variation de l'évacuation gastrique de l'amidon (principe de ce tableau identique à celui du tableau 1).

Sources of variation in the gastric emptying of starch (for explanation, see table 1 ).

\begin{tabular}{|c|c|c|c|c|}
\hline $\begin{array}{l}\text { Terme étudié } \\
\text { Studied term }\end{array}$ & $\begin{array}{l}\text { Loi de VR } \\
\text { Law of } V R\end{array}$ & $\begin{array}{c}\text { Rejet nullité } \\
\text { Nullity rejection }\end{array}$ & $\begin{array}{l}\text { Valeurs de VR } \\
\text { Values of VR } \\
\end{array}$ & $\begin{array}{l}\text { Effets significatifs } \\
\text { Significant effects }\end{array}$ \\
\hline$e_{i}$ & $F(1,8)$ & $V R>5,318$ & 1,854 & - \\
\hline $\mathrm{f}_{\mathbf{j}}$ & $\mathbf{F}(1,8)$ & $\mathrm{VR}>5,318$ & 4,874 & 一 \\
\hline$g_{i j}$ & $\mathbf{F}(1,8)$ & $\mathrm{VR}>5,318$ & 7,588 & $* *$ \\
\hline$e_{i}+g_{i j}$ & F $(2,8)$ & $\mathrm{VR}>4,459$ & 4,721 & $* *$ \\
\hline$f_{j}+g_{i j}$ & $F(2,8)$ & $V R>4,459$ & 6,231 & $* *$ \\
\hline $\mathrm{e}_{\mathrm{i}}+\mathrm{f}_{\mathrm{j}}+\mathrm{g}_{\mathrm{i}, \mathrm{i}}$ & $\mathbf{F}(3,8)$ & $\mathrm{VR}>4,066$ & 4,772 & $* *$ \\
\hline $\mathrm{c}_{\mathrm{ll}}$ & F $(4,32)$ & $V R>2,670$ & 267,368 & $* *$ \\
\hline $\mathrm{m}_{\mathrm{irl}}$ & F $(4,32)$ & $V R>2,670$ & 1,172 & 一 \\
\hline $\mathrm{n}_{\mathrm{jl}}$ & $F(4,32)$ & $V R>2,670$ & 3,237 & $* *$ \\
\hline$o_{i j a}$ & $F(4,32)$ & $\mathrm{VR}>2,670$ & 1,436 & - \\
\hline$m_{i d}+o_{i j, l}$ & F $(8,32)$ & $V R>2,250$ & 1,303 & - \\
\hline$m_{i d}+n_{j i}+o_{i j d}$ & $\mathbf{F}(12,32)$ & $\mathrm{VR}>2,070$ & 1,948 & 一 \\
\hline $\mathrm{J}_{\mathrm{ijpd}}$ & F $\left(32,120^{\circ}\right)$ & $\mathrm{VR}>1,540$ & 1,492 & - \\
\hline $\mathbf{I}_{\mathrm{ijp}}+\mathbf{J}_{\mathrm{ijpd}}$ & $F(40,120)$ & $\mathrm{VR}>1,495$ & 2,493 & $* *$ \\
\hline
\end{tabular}




\section{Amidon (tabl. 3)}

Concernant l'évacuation gastrique de l'amidon, les effets principaux de la nature de l'amidon $\left(\mathrm{e}_{\mathrm{i}}\right)$ et de la source de protéines $\left(\mathrm{f}_{\mathrm{j}}\right)$ du régime sont négligeables. Pourtant, l'influence du régime sur les quantités moyennes d'amidon évacué est certaine $\left(\mathrm{e}_{\mathrm{i}}+\mathrm{g}_{\mathrm{ij}}\right.$ ou $e_{i}+f_{j}+g_{i j}$ ). En fait, ce n'est pas la nature de l'un ou l'autre des constituants considérés qui joue, mais bien leur combinaison qui exerce une influence significative $\left(g_{i j}\right)$.

Par ailleurs le décours de l'évacuation, dont rend compte le terme $c_{d}$, n'est guère affecté que par la source de protéines $\left(\mathrm{n}_{\mathrm{jd}}\right)$. Néanmoins, globalement, la nature du régime (ou le type de combinaison amidon-protéine) n'influence pas significativement le profil des courbes d'évacuation de l'amidon $\left(\mathrm{m}_{\mathrm{id}}+\mathrm{o}_{\mathrm{ijd}}\right.$ ou $\left.\mathrm{m}_{\mathrm{id}}+\mathrm{n}_{\mathrm{jd}}+\mathrm{o}_{\mathrm{ijd}}\right)$. Enfin, comme pour les précédentes variables contrôlées, le facteur porc n'affecte que les quantités moyennes d'amidon évacué sans modifier le décours de cette évacuation (courbes moyennes individuelles parallèles à la courbe moyenne générale pour le régime considéré). Cependant quoique les effets respectifs de $\left(m_{i d}+n_{j d}+o_{i j \ell}\right)$ et de $\mathbf{J}_{\text {ijpd }}$ soient individuellement négligeables, la somme de leurs effets (somme d'effets des facteurs régime et porc) ne l'est pas, comme le montre le calcul de VR $\left(\mathrm{m}_{\mathrm{id}}\right.$ $\left.+\mathrm{n}_{\mathrm{jd}}+\mathrm{o}_{\mathrm{ijd}}+\mathrm{J}_{\mathrm{ijpd}}\right) \simeq \mathrm{F}_{(44,120)}=1,88$ (rejet de la nullité de cet effet global pour $\mathrm{VR}>1,47$ ).

D. Matière fraîche, matière sèche et amidon : ajustements sur l'effet durée

Pour ces 3 variables contrôlées justiciables du modèle univariate, il a été procédé à deux types d'ajustement en fonction de la durée postprandiale.

Un ajustement polynômial est réalisé par régression sur les puissances successives $\left(\mathrm{d}, \mathrm{d}^{2}, \mathrm{~d}^{3}, \mathrm{~d}^{4}\right)$ de chacun des termes liés à la durée postprandiale dans le modèle (3), soit les termes $c_{d}, m_{i d}, n_{j d}, o_{i j l}$. On détermine pour chacun d'eux le polynôme de degré minimum qui fournit le meilleur ajustement. Il apparaît ainsi, pour le terme $c_{d}$ exprimant l'effet principal du facteur durée postprandiale, qu'un bon ajustement des quantités de matière fraîche en fonction du temps écoulé après le repas est obtenu avec un polynôme de degré 2 . Pour ce même terme $c_{\hat{d}}$, un ajustement correct des quantités de matière sèche et d'amidon est obtenu avec des polynômes de degré 3 . Cependant, pour chacune des 3 variables considérées, l'ajustement pour le terme $n_{j d}$ correspond à une fonction linéaire du temps, alors que tout ajustement s'avère inutile pour les termes $\mathrm{m}_{\mathrm{i} d}$ et $\mathrm{o}_{\mathrm{ijd}}$. Aussi peut-on valablement exprimer le décours de l'évacuation gastrique en fonction du temps par un polynôme de degré 2 (matière fraîche) ou de degré 3 (matière sèche et amidon). Pour ces 3 variables contrôlées, les données étant considérées indépendantes (du fait de la statistique du rapport de vraisemblance qui a conduit à accepter le modèle univariate), on se trouve donc ramené très précisément à l'ajustement polynômial des données effectué antérieurement (LAPLACE et al., 1981).

Par ailleurs, a été réalisé un ajustement linéaire en fonction de la racine carrée des durées postprandiales $(\sqrt{ } \mathrm{d})$. Là encore, pour les 3 variables étudiées, tout ajustement s'avère négligeable pour les termes $\mathrm{m}_{\mathrm{id}}$ et $\mathrm{o}_{\mathrm{ijd}}$. Par contre, un tel ajustement linéaire des quantités évacuées en fonction de $\sqrt{\mathrm{d}}$ est justifié pour les 2 termes 
$c_{\mathrm{d}}$ et $n_{\mathbf{j d}}$. Aussi ce type d'ajustement est-il susceptible de rendre compte globalement des effets significatifs au travers d'un nombre de paramètres plus faible que dans le cas de l'ajustement polynômial précédent.

\section{E. Cas de la variable azote (tabl. 4)}

Pour la variable azote, il est apparu précédemment que les données recueillies aux divers instants de collecte ne pouvaient être considérées comme indépendantes, et qu'en conséquence la durée postprandiale ne pouvait être prise en compte que comme une dimension dans un modèle multivariate comportant 36 vecteurs de taille 5 :

$$
\overrightarrow{\mathrm{Y}}_{\mathrm{rps}}=\left(\mathrm{a}+\mathrm{b}_{\mathrm{r}}+\mathrm{I}_{\mathrm{rp}}+\mathrm{e}_{\mathrm{s}}+\mathrm{f}_{\mathrm{rs}}+\mathrm{K}_{\mathrm{rps}}\right) 1, \ldots, 5
$$

Dans ce modèle, les variables aléatoires $I_{\mathrm{rp}}$ et $K_{\mathrm{rps}}$ sont supposées indépendantes deux à deux.

L'hypothèse de nullité (effet négligeable) de chacun des termes correspondant aux effets fixes est ici testée par la statistique du rapport de vraisemblance $L$ qui suit une loi de Wilks ( $\Lambda$, Lambda de Wilks). Il apparaît que l'effet principal du régime $\left(b_{r}\right.$, couple amidon-protéine) est négligeable. Par ailleurs le second terme représentant un effet fixe incluant le régime $\left(\mathrm{f}_{\mathrm{rs}}\right)$ s'avère également négligeable. Si l'on teste alors globalement l'effet semaine $\left(e_{s}+f_{r s}\right)$ on constate, comme pour les précédentes variables contrôlées, que cet effet est encore négligeable. Il faut aussi souligner que si l'effet principal du régime $\left(b_{r}\right)$ n'est pas significatif, l'effet d'interaction régime-porc $\left(I_{r p}\right)$ s'avère important. Enfin, si l'on teste globalement l'effet régime $\left(b_{r}+I_{r p}\right)$ celui-ci apparaît significatif. Ceci revient à dire que au total l'effet régime ne peut être considéré comme nul, mais qu'il est en grande partie masqué par l'effet propre à chaque animal. La nullité de l'effet de la semaine ayant été démontrée ci-dessus, on peut simplifier l'écriture du modèle (4) qui devient :

$$
\vec{Y}_{\text {rps }}=\left(a+b_{r}+I_{r p}+K_{\text {rps }}\right) 1, \ldots, 5
$$

avant de décomposer le terme régime $b_{r}$ en trois termes en fonction des deux sousfacteurs amidon et protéine, soit :

$$
\overrightarrow{\mathrm{Y}}_{\mathrm{rps}}=\left(\mathrm{a}+\mathrm{e}_{\mathrm{i}}+\mathrm{f}_{\mathbf{j}}+\mathrm{g}_{\mathrm{ij}}+\mathrm{I}_{\mathrm{j} \mathrm{jp}}+\mathrm{K}_{\mathrm{ijps}}\right) 1, \ldots, 5
$$

Sur ce modèle (5), on procède aux estimations des divers termes à partir desquelles peuvent être construites les courbes moyennes d'évacuation de l'azote pour chaque régime (fig. 2) sur la base des seuls effets fixes soit $\left(a+e_{i}+f_{j}+g_{i j}\right)$.

On peut alors procéder aux tests de nullité des termes résultant de la décomposition du facteur régime (tabl. 4). L'effet principal de l'amidon ( $\mathrm{e}_{\mathrm{i}}$ ) et l'effet d'interaction amidon-protéine $\left(\mathrm{g}_{\mathrm{ij}}\right)$ s'avèrent négligeables. La valeur des statistiques $\mathrm{L}_{\mathrm{i}}$ et $\mathrm{L}_{\mathrm{ij}}$ implique que l'effet global de l'amidon est véritablement nul. Bien que l'effet principal de la protéine $\left(f_{j}\right)$ soit significatif (mais proche du seuil), l'effet global de la source de protéine $\left(f_{j}+g_{i j}\right)$ reste négligeable. Par conséquent, s'il existe bien à l'égard de l'évacuation gastrique de l'azote un effet du régime, semble-t-il lié principalement à un effet de la protéine alimentaire, cet effet régime reste de toute façon minime relativement à l'effet porc. 


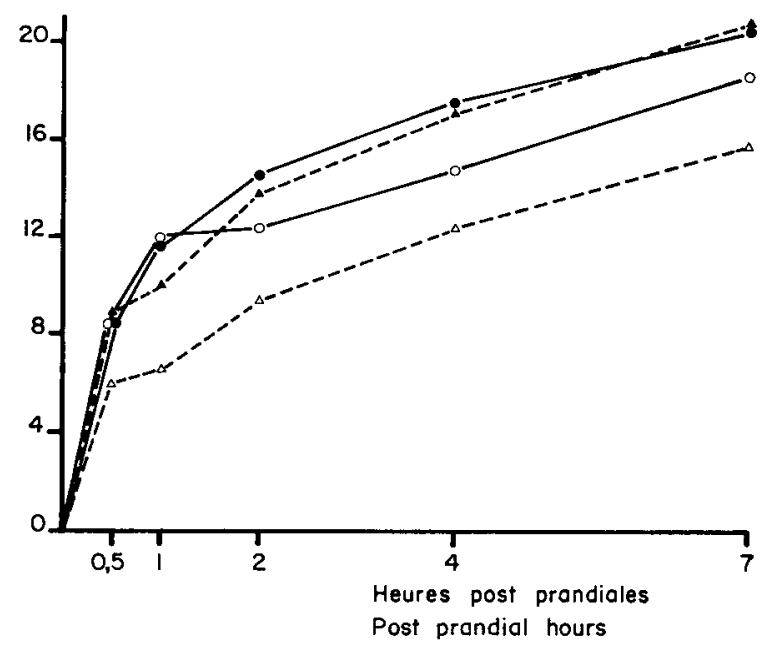

FIG. 2

Courbes moyennes d'évacuation gastrique de l'azote $(g)$ selon la nature du régime.

Ces courbes résultent de l'estimation des divers termes exprimant les seuls effets fixes (amidon, protéine et leur interaction) du modèle (5).

Les symboles sont les mêmes que dans la figure 1 .

Mean gastric emptying curves of nitrogen ( $g$ ) according to the diet.

These curves result from the estimation of the various terms representative of the fixed effects only (starch, protein and their interaction) in the model (5) The symbols used are the same as in figure 1.

\section{TABleaU 4}

Facteurs de variation de l'évacuation gastrique de l'azote.

Sources of variation in the sastric emptying of nitrogen.

\begin{tabular}{|c|c|c|c|c|}
\hline $\begin{array}{c}\text { Terme } \\
\text { étudié } \\
\text { Studied term }\end{array}$ & $\begin{array}{l}\text { Loi de } L \\
\text { Law of } L\end{array}$ & $\begin{array}{l}\text { Rejet de nullité } \\
\text { Nullity rejection }\end{array}$ & $\begin{array}{l}\text { Valeurs de } \mathrm{L} \\
\text { Values of } L\end{array}$ & $\begin{array}{c}\text { Effets } \\
\text { significatifs } \\
\text { Significant } \\
\text { effects }\end{array}$ \\
\hline $\begin{array}{c}\mathbf{b}_{\mathrm{r}} \\
\mathbf{f}_{\mathrm{rs}} \\
\mathbf{e}_{\mathrm{s}}+\mathbf{f}_{\mathrm{rs}} \\
\mathrm{I}_{\mathrm{rp}} \\
\mathbf{b}_{\mathrm{r}}+\mathbf{I}_{\mathrm{rp}} \\
\end{array}$ & $\begin{array}{l}\mathrm{L}_{r} \simeq \Lambda_{\overline{5}} \quad(3,8) \\
\mathbf{L}_{r s} \simeq \Lambda_{\overline{5}} \quad(6,16) \\
\mathrm{L}_{s+r s} \simeq \Lambda_{5} \quad(8,16) \\
\mathrm{L}_{r p} \simeq \Lambda_{5} \quad(8,16) \\
\mathbf{L}_{r+n p} \simeq \Lambda_{\overline{5}}(11,16)\end{array}$ & $\begin{array}{l}\mathrm{L}_{\mathrm{r}} \leqslant 0,0158 \\
\mathrm{~L}_{\mathrm{rs}} \leqslant 0,0610 \\
\mathrm{~L}_{\mathrm{s}+\mathrm{rs}} \leqslant 0,0341 \\
\mathrm{~L}_{\mathrm{rl}} \leqslant 0,0341 \\
\mathrm{~L}_{\mathrm{r}+\mathrm{rp}} \leqslant 0,0161\end{array}$ & $\begin{array}{l}0,0260 \\
0,1864 \\
0,0858 \\
0,0036 \\
0,0039\end{array}$ & $\begin{array}{l}- \\
\overline{-} \\
* *\end{array}$ \\
\hline $\begin{array}{c}\mathrm{e}_{\mathrm{i}} \\
\mathrm{f}_{\mathrm{j}} \\
\mathrm{g}_{\mathrm{ij}} \\
\mathbf{f}_{\mathrm{j}}+\mathrm{g}_{\mathrm{ij}}\end{array}$ & $\begin{array}{l}\mathrm{L}_{\mathrm{i}} \simeq \Lambda_{\bar{j}} \quad(1,8) \\
\mathrm{L}_{\mathrm{j}}=\Lambda_{\bar{j}} \quad(1,8) \\
\mathrm{L}_{\mathrm{ij}} \simeq \Lambda_{\bar{j}} \quad(1,8) \\
\mathrm{L}_{\mathrm{j}+\mathrm{ij}} \simeq \Lambda_{\bar{i}} \quad(2,8)\end{array}$ & $\begin{array}{l}\mathrm{L}_{\mathrm{i}} \leqslant 0,1135 \\
\mathrm{~L}_{\mathrm{j}} \leqslant 0,1135 \\
\mathrm{~L}_{\mathrm{ij}} \leqslant 0,1135 \\
\mathrm{~L}_{\mathrm{j}+\mathrm{ij}} \leqslant 0,0374\end{array}$ & $\begin{array}{l}0,3805 \\
0,0943 \\
0,3742 \\
0,0591\end{array}$ & $\begin{array}{l}- \\
* * \\
-\end{array}$ \\
\hline
\end{tabular}

Valeurs de la statistique $\mathbf{L}$ qui suit une loi de Wilks (Lambda, $\Lambda$ ). La région de rejet de 1'hypothèse de nullité de l'effet considéré est indiquée au seuil de 5 p. 100.

Values of the statistics $L$ approximating the Lambda criterion $(\Lambda)$ of Wilks. The rejection of the hypothesis of nullity of the considered effect is indicated at the level of $5 p .100$. 
Au-delà de cette analyse, l'étude des courbes d'évacuation gastrique de l'azote par l'application du modèle de PotThoff \& Roy (1964) permet de choisir le degré du polynôme représentatif le mieux adapté pour décrire l'évacuation de l'azote en fonction du temps. Il s'avère qu'un polynôme de degré 2 convient dans ce cas; la valeur du rapport de vraisemblance est en effet $\mathrm{L}=0,5704$ pour une région de rejet au seuil 5 p. 100 de $\mathrm{L} \leqslant 0,5301\left(\mathrm{~L} \simeq \Lambda_{2}(4,24)\right)$. Par contre, l'hypothèse d'un ajustement linéaire est rejetée avec $\mathrm{L}=\mathbf{0 , 0 7 1 8}$ pour une région de rejet de $\mathrm{L} \leqslant 0,4151$ ( $\mathrm{L} \simeq \Lambda_{3}(4,24)$. Par ailleurs, l'hypothèse d'un ajustement linéaire par la racine carrée de la durée postprandiale est également rejetée pour la variable azote.

Au total on aboutit donc ici à retenir, comme seule représentation de l'évacuation de l'azote en fonction du temps, un polynôme de degré minimum $2\left(\mathrm{Y}=\mathrm{b}_{0}+\mathrm{b}_{1} \mathrm{t}\right.$ $+b_{2} t^{2}$ ) soit d'un degré moindre que celui (degré 3) antérieurement retenu pour cette même variable (LAPLACE et al., 1981). Toutefois, on remarque la très grande similitude entre les courbes moyennes descriptives de l'évacuation de l'azote (effets fixes, fig. 2) et les profils polynomiaux de degré 3 décrivant cette même évacuation dans le travail antérieur.

\section{Discussion}

Le problème général d'ordre méthodologique que constitue l'approche de la cinétique de phénomènes de transit, tels que l'évacuation postprandiale des contenus gastriques, a été souligné dans l'introduction de ce travail. Cette difficulté tient à l'influence du temps sur la valeur des observations, c'est-à-dire à l'indépendance ou à la non indépendance des données enregistrées consécutivement. C'est en considérant a priori comme une évidence que les quantités recueillies $30 \mathrm{~min} ., 1 \mathrm{~h}, \ldots, 7 \mathrm{~h}$ après le repas ne sont pas indépendantes entre elles, qu'une procédure d'analyse multidimensionnelle a été antérieurement mise en œuvre (LAPLACE et al., 1981) pour prendre en compte le temps comme une dimension et non comme un facteur. Or il ressort de ce qui précède que non seulement le facteur temporel de répétition d'une semaine à l'autre est nul pour chacune des 4 variables contrôlées, mais aussi que la valeur des observations réalisées à chaque instant de la cinétique est indépendante des facteurs temporels parasites jour et durée postprandiale pour les variables matière fraîche, matière sèche et amidon. L'intérêt évident de ce fait, et des tests qui légitiment donc l'usage du modèle univariate pour ces 3 variables, est de permettre de conduire l'analyse sur 180 données (observations), et non sur 36 données seulement (vecteurs) comme l'exige la dépendance conduisant à retenir le modèle multivariate dans le cas de l'azote. Ceci signifie que vérifier l'indépendance des observations consécutives conduit à accroître notablement la puissance de l'analyse du seul fait de l'augmentation (5 fois) du nombre de données. Toutefois, l'avantage d'indépendance des observations ici mis à profit pour 3 variables ne peut être considéré que comme circonstanciel. On ne saurait généraliser a priori sans tester la validité éventuelle du modèle univariate pour chaque expérience. La vérification a d'autant plus d'intérêt que l'on peut à l'inverse envisager que la dépendance dans le cas de l'azote résulte de la présence dans les contenus digestifs d'une proportion non négligeable d'azote endogène.

Par ailleurs, le travail présenté ici permet d'identifier très clairement l'influence respective de chacun des termes du modèle et son rôle particulier dans la définition 
du profil d'évacuation gastrique enregistré. Il autorise par là même non seulement une révision des effets de l'amidon et des protéines alimentaires mais aussi, lorsque ceux-ci sont confirmés, une meilleure compréhension de ces effets. Ainsi, il est vérifié que l'évacuation de la matière fraîche est affectée par la source de protéines du régime, cette influence étant elle-même toujours significativement modulée par la nature de l'amidon associé. Cette conclusion déjà retenue antérieurement (LAPLACE et al., 1981) ne concerne cependant que le niveau moyen d'évacuation. On peut de surcroît préciser que le profil des courbes, c'est-à-dire le décours de l'évacuation de la matière fraîche pour chaque régime, n'est affecté que par la source de protéines ce qui implique le parallélisme des courbes représentatives des régimes comportant une même protéine, en accord avec les résultats des tests effectués antérieurement sur les profils polynômiaux (Laplace et al., 1981). Enfin, le fait que l'influence de l'animal se traduise par un simple effet de translation des courbes moyennes, signifie que le niveau moyen d'évacuation d'un régime donné peut varier d'un porc à l'autre sans modification du profil ou décours de l'évacuation. Ceci conduit à souligner l'importance du nombre de porcs mis en cuvre pour évaluer ce qu'est réellement, en moyenne, l'évacuation gastrique d'un régime donné.

Cette distinction entre les effets sur le niveau moyen d'évacuation et ceux qui concernent son décours, amène à modifier assez largement les conclusions relatives à l'évacuation de la matière sèche. En effet, il n'y a pas d'effet régime significatif sur le niveau moyen d'évacuation de la matière sèche des 4 régimes testés, alors que le profil de la courbe dépend de la nature de l'aliment ingéré et plus spécialement de la source de protéines alimentaires. Cette dernière particularité confirme encore le parallélisme des courbes d'évacuation de la matière sèche des régimes contenant une même protéine (LAPlace et al., 1981). Par ailleurs, le fait que le régime influence la valeur moyenne d'évacuation de la matière fraîche et pas celle de la matière sèche, pourrait être relié à l'absence de correction des mesures pour les volumes d'eau d'origine secrétoire. Si l'appétibilité des régimes entraîne des différences non négligeables des sécrétions salivaire et gastrique, toute augmentation particulière de celles-ci conduit à surestimer la quantité résiduelle donc à sous-estimer la quantité évacuée de matière fraîche. Aussi, les mesures réalisées ne permettent-eiles pas d'écarter l'hypothèse que l'effet régime sur la matière fraîche soit artefactuel, quoique lié à un phénomène physiologique. Par contre, le déroulement de l'évacuation est, pour la matière fraîche comme pour la matière sèche, significativement influencé par la source de protéines alimentaires.

Sur un plan plus général, l'absence de différence due au régime des quantités de matière sèche évacuées en moyenne pourrait être reliée à la similitude des caractéristiques physiques des régimes purifiés utilisés, dont l'évacuation ne requiert, pour aucun d'eux, une action de broyage prolongée par l'antre gastrique. Mais, si l'évacuation est en moyenne la même au cours des 7 heures postprandiales, son déroulement n'est pas le même, probablement par l'intervention des facteurs liés à la composition chimique (en l'occurrence surtout à la source de protéines) agissant essentiellement sur les résistances à l'écoulement. En fait, la dualité d'effet de la nature du régime sur l'évacuation gastrique de la matière sèche, telle qu'elle ressort de ce travail, coïncide avec la conception actuelle de l'hydraulique gastroduodénale (LAPlace, 1982). Mais cette dualité signifie aussi que deux régimes pour lesquels des quantités de matière sèche en moyenne équivalentes sont évacuées dans un délai donné, peuvent fort bien être digérés (hydrolyses-absorption) de façon très différente : en effet la différence de déroulement de l'évacuation peut conduire en 
dernier ressort à modifier les cinétiques d'apport des nutriments à l'organisme. Par conséquent, il est nécessaire de disposer d'un nombre suffisant d’animaux pour évaluer correctement l'évacuation gastrique moyenne, l'effet de translation dû à l'influence de l'animal étant observé également pour la matière sèche; mais il est non moins nécessaire de prendre en compte de façon complète la cinétique du phénomène c'est-à-dire de ne pas se borner à un bilan d'évacuation à tel ou tel temps posprandial.

Dans le cas de l'amidon, le niveau moyen d'évacuation gastrique est significativement affecté par le seul effet de la combinaison particulière d'un amidon et d'une protéine. Dans la mesure où l'amidon constitue environ 70 p. 100 de la matière sèche ingérée, cet effet d'interaction important pourrait être à l'origine de l'effet significatif d'interaction également enregistré pour la matière sèche, quoiqu'insuffisant dans ce dernier cas pour entraîner un effet régime global. Quoi qưil en soit, l'association particulière d'un amidon et d'une protéine, lors de la formulation d'un régime, affecte de façon significative le niveau moyen d'évacuation de l'amidon par l'estomac. Ce fait implique donc un étalement plus ou moins grand dans le temps de la digestion du repas. A ce phénomène important s'ajoutent, comme pour la matière fraîche et la matière sèche, un effet significatif de la source de protéines sur le déroulement de l'évacuation de lamidon et une variation, par trans!ation de la courbe, liée à l'influence du porc.

En somme, l'analyse ici réalisée pour les trois variables contrôlées justiciables d'un modèle univariate, conduit à confirmer un certain nombre des conclusions antérieures (LAPLACE et al., 1981) relatives à l'importance de la source de protéines, de l'interaction amidon-protéines, et de l'influence du porc. Mais elle amène aussi à une rectification dans la mesure où n'existe pas en réalité, pour aucune des variables, d'effet principal lié à la nature de l'amidon purifié mis en œuvre. Il semble que ce soit la seule conséquence de l'introduction abusive de l'effet porc comme un facteur dans le modèle d'analyse antérieurement mis en ouvre (LAPLACE et al., 1981), ce qui a donc effectivement conduit à surestimer l'effet amidon. Mais au-delà de cette rectification, le présent travail permet aussi de préciser la nature des effets. Ceux-ci peuvent être résumés comme suit, de façon à en tirer les conséquences quant au déroulement de la digestion :

(i) Pour les trois variables étudiées (matière fraîche, matière sèche et amidon) et pour un niveau moyen donné d'évacuation, le déroulement de cette dernière (traduit par le profil de la courbe), c'est-à-dire la cinétique du phénomène, est toujours significativement influencé par la source de protéines du régime. Ceci revient à dire que les protéines jouent un rôle décisif à l'égard de la chronologie de l'évacuation et de la cinétique de mise à disposition ultérieure des nutriments.

(ii) L'association particulière d'un amidon et d'une protéine affecte par contre le niveau moyen d'évacuation de l'amidon, effet semble-t-il partiellement répercuté sur la matière sèche. Ceci signifie que c'est le type de couple amidon-protéine présent dans l'aliment qui influence principalement la durée globale de digestion. En effet, indépendamment de l'aspect cinétique à l'intérieur d'un intervalle de temps donné, un faible niveau moyen d'évacuation implique un temps total d'évacuation plus long pour une même quantité ingérée d'amidon. Les facultés d'hydrolyse enzymatique et d'absorption étant en général sollicitées en deçà de leur limite supéricure (RERAT, 1975 ; RERAT et al., 1977), le temps total de digestion d'une quantité donnée d'amidon, qui peut être évalué par l'annulation des différences porto-artérielles des concentrations de sucres réducteurs (RERAT, 1975) serait déterminé par l'association dans l'aliment de tel amidon à telle protéine. 
(iii) Limportance de linfluence du porc a déjà été soulignée. Compte tenu de la contrainte expérimentale fréquente d'affectation des animaux à un seul régime, il est évident qu'un nombre trop réduit de sujets expérimentaux peut conduire, selon les cas, à «identifier» un effet régime inexistant ou à l'inverse à masquer un effet régime bien réel. De fait, le phénomène de translation dû à l'influence $d u$ porc ne fausse pas l'appréciation du déroulement du phénomène, mais peut fausser celle du niveau moyen d'évacuation.

Le risque inhérent à l'usage d’un nombre réduit d'animaux pour l'étude de phénomènes de transit a déjà été souligné par MAC RAE (1974) chez le mouton : la variabilité des mesures étant plus d'origine interindividuelle que intraindividuelle, elle doit être considérée comme «un authentique phénomène biologique », ce qui conduit MAC RAE (1974) à mettre en question les nombreux travaux nutilisant que 2 ou 3 animaux fistulés. Le problème de l'influence de l'animal s'avère d'autant plus important que le nombre d'observations est réduit. L'exemple en est apporté par l'étude de la variable azote pour laquelle la nécessité de mise en ouvre d'un modèle multivariate réduit le nombre de données. Pour cette variable, il apparaît en effet que, globalement, l'effet régime est pour l'essentiel masqué par l'effet porc. En d'autres termes, dans cette expérience qui a mis en ouvre 12 porcs, soit 3 par régime, la variation entre individus est plus importante que celle due à la source de protéines alimentaires. Ceci implique que le nombre de porcs mis en œuvre est insuffisant pour l'évaluation de l'effet régime sur l'évacuation gastrique de l'azote par analyse multivariate. Cette affirmation est étayée a contrario : pour les variables matière fraîche, matière sèche et amidon, l'usage licite du modèle univariate a permis de mettre en évidence divers effets statistiquement significatifs; l'utilisation pour ces 3 mêmes variables du modèle multivariate, tel qu'appliqué à la variable azote, ne permet pas à ces mêmes effets d'être statistiquement significatifs.

Dans ces conditions, quel est l'intérêt des procédures d'ajustement ? L'ajustement linéaire des quantités évacuées en fonction de la racine carrée des durées postprandiales pourrait rendre compte globalement des effets pour trois des variables étudiées, mais n'est pas utilisable dans le cas de l'azote. Par contre, l'ajustement polynomial des données, appliqué de longue date à l'évacuation gastrique (LAPLACE \& TomasSONE, 1970) est satisfaisant pour toutes les variables contrôlées. En effet, l'ajustement polynômial reste utilisable pour la variable azote, le degré retenu (trois ou deux) n'ayant pas en définitive d'incidence véritable sur les tests de parallélisme et d'égalité et les déductions qui ont pu en être tirées. Globalement, l'ensemble de ces dernières coïncide avec les effets identifiés comme significatifs dans ce travail pour les 4 variables contrôlées. De plus, la similitude flagrante des profils polynômiaux antérieurement présentés (LAPLACE et al., 1981) et des courbes moyennes exprimant les effets fixes (fig. 1 et 2), montre que l'ajustement polynômial des données (et les tests de parallélisme et d'égalité qui s'y rattachent) constitue un bon outil de travail pour l'expérimentateur. La seule précaution tient au fait que, si l'ajustement polynômial peut être réalisé de façon simple lorsque l'indépendance des observations est vérifiée, la dépendance de ces dernières (cas de l'azote) impose l'usage d'un ajustement par le modèle de Potтhoff \& Roy (1964). Ceci étant, l'ajustement polynômial permet de visualiser des phénomènes qui ne s'expriment pas nécessairement en termes significatifs dans une analyse de variance, soit en raison des conditions particulières de l'expérience, soit du fait d'un nombre restreint d'animaux. 


\title{
V. Conclusions
}

Il ressort de cette étude analytique des effets de la nature de l'amidon et de la source de protéines à l'égard de l'évacuation gastrique : (i) que la nature de l'amidon est en elle-même sans influence dans le cas des amidons purifiés (de maïs ou de blé) utilisés; (ii) que lassociation particulière d'un amidon à une protéine affecte le niveau moyen d'évacuation de la matière fraîche et de l'amidon, mais que celui de la matière sèche reste exempt de tout effet du régime, (iii) que la source de protéines du régime influence fortement le déroulement de l'évacuation de la matière fraîche, de la matière sèche et de lamidon.

Il savère très important de bien distinguer le niveau moyen d'évacuation, qui peut s'exprimer par un bilan d'évacuation au terme d'un laps de temps donné, de la cinétique du phénomène par lequel est réalisé ce résultat au cours de l'intervalle considéré. Il semble en effet que l'association particulière d'un amidon et d'une protéine, affectant le niveau moyen d'évacuation détermine par là même la durée globale de digestion du repas; par contre les protéines joueraient un rôle décisif à l'égard du déroulement de l'évacuation et de ce fait à l'égard de la cinétique de mise à disposition ultérieure des nutriments.

Enfin, l'influence de l'animal est considérable dans la mesure où elle s'exerce sur le niveau moyen d'évacuation d'un régime donné sans altération du profil de la courbe, et où l'importance de cette variabilité entre individus peut aisément masquer les effets expérimentaux comme c'est ici le cas pour l'azote. Au plan méthodologique, ceci souligne la nécessité d’un nombre d'animaux et d'observations plus élevé que celui habituellement mis en ouvre dans ce type d'expérimentations.

\section{Accepté pour publication en juin 1983.}

\author{
Summary \\ Revision of the effects of dietary sources of starch and protein \\ on gastric emptying in the pig
}

Gastric emptying of 4 diets (16 p. 100 crude protein) resulting from the factorial combination of 2 purified starches (maize or wheat) and 2 protein sources (fish meal or wheat gluten) has been described previously (LAPLACE et al., 1981) on the basis of the residual gastric contents at various post prandial times in 12 pigs fitted with a gastric cannula. However, the statistical study of the emptied quantities of wet chigesta, dry matter, starch and nitrogen was questioned. The nullity of the influence of the repetition of measurements from week to week was demonstrated for the 4 controlled variables. In addition, for 3 of them (nitrogen excepted), the values recorded at the consecutive sampling times after the meal were shown to be independent of time related factors. Therefore, the analyses of variance used were univariate for wet digesta, dry matter and starch, and multivariate for nitrogen.

Results showed that (i) the kind of purified dietary starch did not significantly affect gastric emptying; (ii) the particular combination of one starch with one protein influenced the mean gastric emptying of wet digesta and starch, while there was no significant effect of the diet on dry matter emptying; (iii) the dietary protein source strongly influenced the 
pattern of emptying. The importance of such a distinction between the effects on the mean rate of emptying and those on the kinetics of emptying is stressed. Any particular starchprotein association would affect the mean rate of emptying and thus the total duration of the digestion, while proteins would determine the pattern of emptying and thus the kinetics of further nutrient delivery. At last, a strong influence of the animal was demonstrated which hinders from identifying the dietary effects on nitrogen emptying.

\section{Références bibliographiques}

Darcy B., Laplace J.P., Villiers P.A., 1981. Digestion dans l'intestin grêle chez le porc. 4 - Cinétique de passage des digesta au niveau de la jonction iléo-caeco-colique et bilans de la digestion selon la nature de l'amidon et la source de protéines alimentaires. Ann. Zootech., 30, 31-62.

GiLl J.L., Hafs H.D., 1971. Analysis of repeated measurements of animals. J. anim. Sci., 33, 331-336.

Krishnaiah P.R., 1969. Multivariate analysis of variance and related topics, 55-168. In : Multivariate Analysis, part II, 1 vol., Academic Press, New York.

LaPlace J.P., 1982. Fonction de l'estomac chez le pore : révision des concepts usuels sur son rôle dans la digestion et la mécanique de son évacuation, 29-44. In : LAPLACE J.P., Corring T., Rerat A., Physiologie digestive chez le porc, I.N.R.A. Publ. Ed. (Les Colloques de l'I.N.R.A., $\mathrm{n}^{\circ}$ 12).

Laplace J.P., Darcy B., Pons O., 1983. Digestion dans l'intestin grêle chez le porc. 5 - Importance de la variabilité non contrôlée; modélisation des profils moyens de transit iléo-caeco-colique par régression polynomiale. Ann. Zootech., 32, 201-214.

Laplace J.P., Pons O., Cuber J.C., Kabore C., Villiers P.A., 1981. Effets de la nature de l'amidon (blé ou maïs) et des protéines (poisson ou gluten) sur les facteurs de contrôle et le décours de l'évacuation gastrique d'un régime semi-purifié chez le porc. Applications de l'analyse multidimensionnelle et de la régression polynomiale. Ann. Zootech., 30, 209-248.

Laplace J.P., Tomassone R., 1970. Evacuation gastroduodénale chez le porc; fistulation chronique par voie thoracique extrapleurale; recherche d'une technique d'analyse mathématique de l'évacuation. Ann. Zootech., 19, 303-332.

MAC RAE J.C., 1974. The use of re-entrant cannulae to partition digestive function within the gastrointestinal tract of ruminants, 261-276. In : MCDonald I.W., WARNER A.C.I., Digestion and metabolism in the ruminant. Proc. 4th Symp. Ruminant Physiol., Sydney.

Potthoff R.F., RoY S.N., 1964. A generalized multivariate analysis of variance model useful especially for growth curve problems. Biometrika, 51, 313-326.

Rerat A., 1975. Mesure quantitative in vivo de l'absorption chez le porc. Application aux sucres et aux acides aminés, 47-62. In : Réanimation entérale à faible débit continu, I.N.S.E.R.M. Ed. (Les Colloques de l'I.N.S.E.R.M., vol. 53).

Rerat A., Corring T., Vaissade P., Vaugelade P., 1977. Postprandial absorption of $\alpha$-amino nitrogen and sugars in pigs. Quantitative measurement using discontinuous pancreatic derivation. Ann. Biol. anim. Biochim. Biophys., 17, 583-588.

SCHEFFE H., 1967. The analysis of variance, 1 vol., Wiley Ed., New York. 